\title{
Effects of Applying Virtual Reality for Immersive Anxiety Reduction in Dental Patients
}

\author{
Seiya Liu BA ${ }^{1}$, Annabelle NG ${ }^{2}$, Maya Cratsley ${ }^{3}$ and Ardini Tandya DDS ${ }^{4 *}$ \\ ${ }^{1}$ University of Miami, Miller School of Medicine, Miami, 33130, Florida, USA \\ ${ }^{2}$ Georgetown University, 3700 O St NW, 20057, Washington DC, USA \\ ${ }^{3}$ Brown University, B.S. Providence, 02912, Rhode Island, USA \\ ${ }^{4} 8950$ W Olympic Blvd \# 208, Beverly Hills, 90211, California, USA
}

*Corresponding author: Ardini Tandya DDS, 8950 W Olympic Blvd \# 208, Beverly Hills, CA 90211, USA, Tel: +1 (424) 535 3123, E-mail: marinmomo@gmail.com

Received: October 21, 2019; Accepted: November 04, 2019; Published: November 12, 2019

\begin{abstract}
Background: There is a lack of cost-effective solutions to improve mental wellness, anxiety, for anyone seeking medical or dental procedures, chemotherapy, or even MRI. Not much data exists on mental wellness solutions for patients, either preor post- procedure. In addition, uncontrolled anxiety can lead to physiological side effects of tachycardia, excessive bleeding, pro-longed procedure, care avoidance and decreased health. Prior research has shown some success using Virtual Reality interventions for improving anxiety disorders. This study further examined an innovative VR relaxation intervention aiming to decrease anxiety in dental patients.

Methods: After IRB approval 20 dental patients participated in the study. Participants completed a 10-item STAI questionnaire and then spent 10-15 minutes immersed in a relaxing virtual environment. Participants then filled out another 10-item STAI questionnaire, the Corah's Dental Anxiety questionnaire, and a user experience questionnaire.

Results: There was a high significant decrease in anxiety between pre-intervention $(\mathrm{M}=18.53$, $\mathrm{SD}=4.29)$ and post-intervention $(\mathrm{M}=15.63, \mathrm{SD}=3.63)$ measures $(\mathrm{p}<0.01)$. User acceptance was high, with a large majority of patients reporting feeling relaxed and wanting to use the virtual reality technology again.

Conclusions: These results suggested the novel VR wellness technology can be very effective at reducing pre-dental procedure anxiety in patients. This intervention can be beneficial in the field of anxiety reduction.
\end{abstract}

Keywords: Dental anxiety; Anxiety; Virtual reality; Anxiety reduction using virtual reality; Mental wellness

\section{Introduction}

Anxiety has been extensively studied in the mental health and wellness fields. Prominent theories have separated anxiety into a "state" dimension-anxiety at the present moment-and a "trait" dimension, which refers to dispositional anxiety [1-3]. Dental

Citation: Seiya Liu BA, Annabelle NG, Cratsley M, et al. Effects of Applying Virtual Reality for Immersive Anxiety Reduction in Dental Patients. J Anxiety Depress. 2019;2(2):117. 
www.yumedtext.com | November-2019

anxiety (DA) is a specific form of trait anxiety associated with the thought of visiting the dentist for preventive care or dental procedures [4]. Anywhere from 50\% to $80 \%$ of adults in the United States experience mild to severe dental anxiety [5]. Higher DA leads to avoidance of dental care, as well as deteriorated quality of life and oral health in adult patients [6]. Patients with DA visit the dental office only in acute emergency situations. They must receive intensive and traumatic treatment procedures, which further reinforces their DA and leads to complete avoidance of care. Consequently, patients with mismanaged DA enter a vicious cycle of DA, delayed visiting, dental complications, and painful treatment. Thus, DA is widely considered a significant challenge for both patients and providers in oral healthcare. There remains a great need for the development of an effective alternative to analgesic interventions for DA, which are costly and often accompanied by side-effects [4].

There is growing evidence that virtual reality (VR) has the potential to greatly improve various aspects of mental health and well-being. The immersive and interactive nature of VR can be employed to improve existing mental health interventions because it gives users increased sensory input and a sense of "presence," which help decrease distressing thoughts and emotions [7]. VR has been used to enhance the treatment of generalized anxiety, substance use disorders, and other psychiatric disorders in various non-dental populations [8]. A recent review found promising results for the effectiveness of VR in decreasing stress, reducing distress, and improving mood [9]. For example, Yu et al. immersed healthy participants in either an urban environment or a natural, forest environment. While the urban environment had no effect on mood, the natural environment significantly reduced self-reported fatigue, tension, and depression [10]. Similarly, Riva et al. used an immersive, interactive virtual park system designed to elicit either anxiety (through darkness and ominous sounds) or relaxation (through natural light and relaxing sounds). The environments successfully induced both self-reported anxiety and relaxation [11].

Despite the high prevalence of DA in the United States, and the proven effectiveness of VR, researchers have devoted insufficient attention to implementing VR in order to reduce dental anxiety. The majority of studies have focused on using VR as a distraction from pain during dental procedures [12-15]. Tanja-Dijkstra et al. evaluated the effectiveness of a VR environment of a coastal nature area to distract participants during simulated dental treatment. The VR distraction reduced the vividness of dental patients' memories a week after the procedure, but no measures were taken regarding mental state or anxiety [16]. Several studies in recent years, however, have supported the efficacy of VR exposure therapy in the treatment of dental phobia, a condition related to DA [17-19].

The VR relaxation intervention employed in this study has previously been tested on cancer patients and student athletes. After being immersed in VR-R, 92\% of the sample of cancer patients felt relaxed, 90\% experienced a reduction in anxiety, and $88 \%$ felt reduction in fear [20]. The intervention also had positive effects on student athletes, with $75 \%$ reporting that they felt relaxed and $90 \%$ stating that they would use it again [21]. The VR-R intervention has been shown to be effective in inducing relaxation and reducing negative mental conditions but has not yet been assessed in a dental context. Lin, Wu, and Yi have established a strong association between trait dental anxiety and state anxiety during dental visits, and there is evidence that assessing state anxiety is critical to managing anxiety for high-DA patients, as well as pain control for all dental patients [6]. Thus, state anxiety (SA) is an effective measurement for changes in anxiety levels prior to dental procedures. 
The present study aims to determine the effects of a pre-treatment VR relaxation (VR-R) intervention on subjective relaxation and state anxiety (SA) in dental patients.

\section{Methods}

\subsection{Study review}

The study was reviewed and approved by the Western Institutional Review Board (Western IRB) on June $6^{\text {th }}$, 2019. Study Number: 1260154. IRB Tracking Number: 201191357.

\subsection{Participants}

Twenty-one adult dental patients receiving treatment from the Dr. Tandya's dental office in Beverly Hills, California were recruited in person during the month of July 2019. One participant dropped out, resulting in a sample size of $n=20$ total participants. Of the total participants, 9 were Male, 10 were Female, and 1 did not indicate their gender. Participant ages ranged from 20-66, with an average age of 41. Patients who were over 18 years old, had normal or corrected to normal vision, and provided written informed consent were selected for participation and compensated with a $\$ 30$ gift card. Patients with pre-existing history of seizures and/or who did not take medication for their seizures were excluded from the study.

\subsection{Equipment}

The Oculus Go headset with head tracking technology was utilized to deliver the VR-R intervention. Users were asked to spend 10-15 min in a virtual environment (VE) in the WORLDS application, which is designed by IFGworld" ${ }^{\mathrm{TM}}$. The "Onsen" environment was chosen for this study due to historical and scientific evidence that hot springs have relaxing, therapeutic properties [22,23]. The VE depicted a hot spring, with a sunset and falling snow, populated by trees and an interactive rubber duck. 360-degree audio of rushing water also functioned as a primary feature of the VE (FIG. 1).

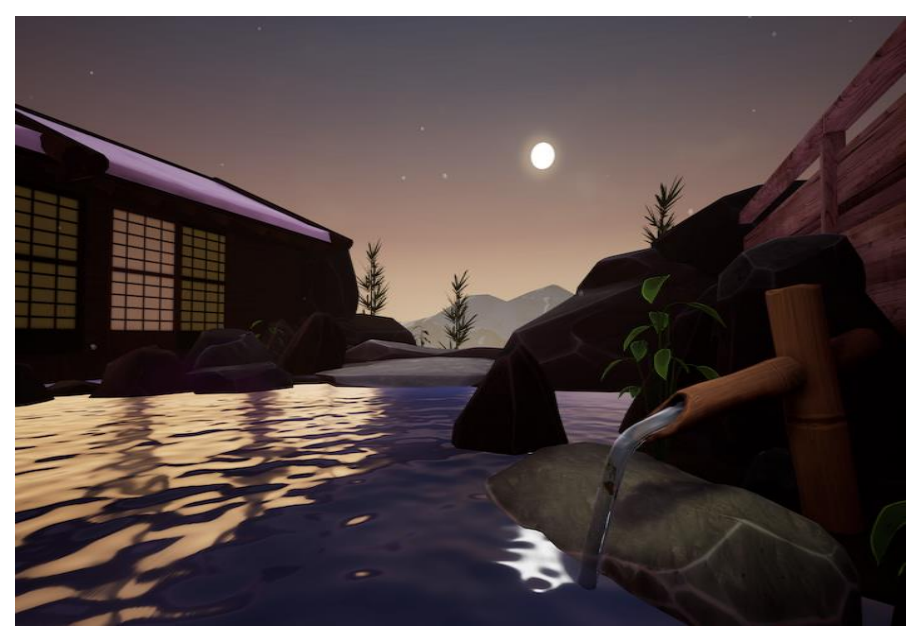

FIG. 1. The virtual environment used in the study. This is the "Onsen" environment from the VR app WORLDS by IFGworld. 


\subsection{Study procedure}

All subjects were taken through the informed consent process prior to the study. A within-subjects design was employed to assess the effectiveness of the VR intervention in reducing state anxiety and increasing relaxation before receiving dental treatment. Volunteers completed a set of pre-intervention questionnaires that included demographic data and the first 10 items from the State-Trait Anxiety Inventory for Adults (STAI). Volunteers then received the VR-R intervention while sitting in a chair in the waiting area of the dental office for 10 minutes (FIG. 2). Afterwards, post-intervention questionnaires were administered consisting of the last 10 items from the STAI, Corah's Dental Anxiety Scale, as well as subjective user experience questions.

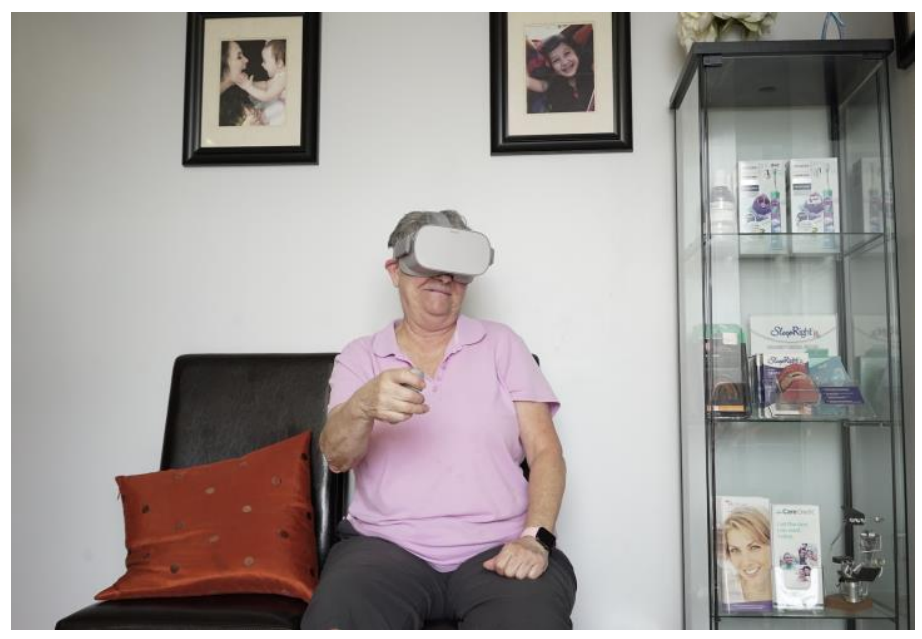

FIG. 2. The participant uses the VR for 10-15 minutes while sitting and waiting to be called for dental treatment.

\subsection{Data coding}

For cases in which participants circled two adjacent answers, the response was assumed to be in between those two answers, and thus the mean was taken. Overall scores were obtained for the dental anxiety scale based on the scoring guidelines. The guidelines for the State Anxiety scale were used to obtain pre-intervention and post-intervention anxiety scores ranging 1040 , as opposed to the traditional range of 20-80 for the full scale.

\section{Results}

19 participants completed all portions of the survey. One participant did not complete the pre-intervention STAI questionnaire due to experimenter error. This participant's data was excluded from the State Anxiety analysis ( $\mathrm{n}=19)$ but included for all other analyses $(n=20)$. Of the 20 participants, $80 \%$ of participants indicated that the VR intervention helped them relax or reduce their anxiety/negative feelings prior to the dental procedure and $75 \%$ said that they would want to use IFGworld VR environments the next time they came to the dentist's office, 75\% said the technology made their experience more relaxing than usual, and $75 \%$ indicated that they would continue to use the VR technology after their visit for the purposes of general wellness. $75 \%$ reported no adverse effects. $20 \%(\mathrm{n}=4)$ experienced mild nausea, and $5 \%(\mathrm{n}=1)$ selfreported an adverse effect of "confusion/disorientation". With regards to dental anxiety, 35\% of participants scored low for 
www.yumedtext.com | November-2019

DA $(n=7), 40 \%$ showed moderate DA $(n=8), 5 \%$ showed high DA $(n=1)$, and $15 \%$ showed severe DA). On the user experience survey, $85 \%(n=17)$ reported that the app was easy to navigate, and $90 \%(n=19)$ reported that the controls were easy to understand.

A paired two-tailed t-test was performed excluding the participants who experienced adverse effects $(n=15)$ and there was a highly significant difference between pre-intervention $(\mathrm{M}=18.53, \mathrm{SD}=4.29)$ and post-intervention $(\mathrm{M}=15.63, \mathrm{SD}=3.63)$ measures $(\mathrm{t}(14)=-3.19, \mathrm{p}<0.01,95 \% \mathrm{CI}=-4.85,-0.95)(\mathrm{FIG} .3)$. A two-tailed paired t-test was performed comparing preintervention anxiety scores to post-intervention anxiety scores for all participants. There was not a significant difference between anxiety levels in the pre-intervention $(\mathrm{M}=18.26, \mathrm{SD}=4.33)$ and the post-intervention $(\mathrm{M}=16.45$, $\mathrm{SD}=4.32)$ measurement periods $(\mathrm{t}(18)=-1.52, \mathrm{p}=0.15,95 \% \mathrm{CI}=-4.32,0.692)$. However, there was a general trend towards decreased anxiety.

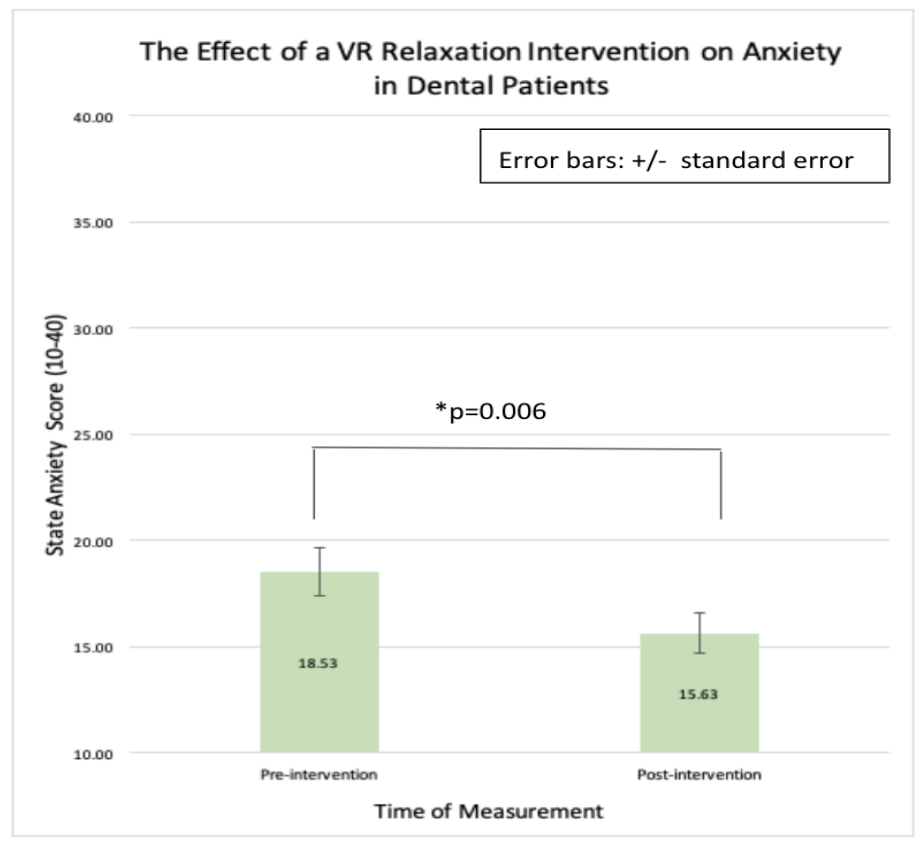

FIG. 3. The effect of VR-R intervention on anxiety (for participants without adverse effects).

Of the participants who experienced adverse effects, one showed a decrease, two showed an increase, and one showed no change in state anxiety levels. The average DA score for participants who had adverse reactions to the VR was 13.00 , compared to 9.26 for those who did not experience adverse effects, and 10.20 for the sample overall. $33 \%$ of the total participants with severe DA scores $(n=1)$ saw a decrease in anxiety and no adverse effects.

\section{Discussion}

There is a lack of cost-effective tools to improve mental wellness in the healthcare facilities, specifically to help patients cope with anxiety pre-procedure. Many studies have shown the potential of virtual reality to improve anxiety, but the costs of such technology have always been challenged. In the past the cost of head mount and software was not effective for scalable 
www.yumedtext.com | November-2019

treatments. Current advancement in using mobile VR light weight headset and the development of app in the head mount has created a great opportunity to scale product and decrease costs. Current study used the mobile Oculus Go and the software from Worlds ${ }^{\mathrm{tm}}$ app in the Oculus store. The Worlds ${ }^{\mathrm{tm}}$ was created from many environments for relaxation. Current study used "Onsen" environment to be consistent for all subjects. The data from this study illuminates the benefits of this particular VR$\mathrm{R}$ intervention for pre-dental procedure for patients. Given the demonstrated possibility of adverse effects such as nausea and disorientation, this intervention should be administered with care and caution. The data gives the impression of a relationship between high/severe Dental Anxiety scores and the likelihood of experiencing adverse effects in VR. The participants who experienced adverse effects had on average higher DA scores; however, one out of three participants with a "severe" DA score did not experience any adverse effects and experienced anxiety reduction and relaxation benefits. Due to the conflicting data and very small sample size, more research is required to make confident claims about the effects of this intervention on individuals with high or severe DA. Future studies should screen for patients with high or severe DA scores to further explore the risks and benefits of this technology for higher DA populations.

This intervention provided anxiety reduction benefits to the vast majority of participants. While the reduction in anxiety was not quite statistically significant for the population as a whole, it was highly significant among the participants who did not experience adverse effects. Experiencing adverse effects appears to, reasonably, take away from the benefits of the intervention. Additionally, user acceptance was relatively high for this intervention, with a large majority of patients selfreporting feeling relaxed and wanting to use the technology again in a dental or non-dental setting. This provides impressive evidence for the effectiveness of this intervention.

The use of VR in clinical settings for anxiety reduction is still relatively new, and more testing is required to better understand the risks and benefits of using this technology. This study offers a strong case for the capacity of the IFGworld VR relaxation application to reduce pre-dental procedure anxiety and aid in relaxation for patients in a waiting room setting. If patients' pre-procedure anxiety is reduced through the use of VR-R, patients may have less side effects and complications during and after the dental procedure. In addition, he or she can be less avoidant of seeking dental care, leading to improvements in oral health and quality of life overall for patients [6].

This intervention could thus benefit patients' overall health, also benefiting the dental offices that utilize the intervention by increasing the number of appointments and decreasing cancellations. Future study should expand patient's population, uses of different VR environments, and explore the generalizability of these findings to other clinical applications, examining factors such as preoperative anxiety or illness-related anxiety in other patient population.

\section{Conflict of Interest Statement}

The authors whose names are listed immediately below report affiliation and involvement with the following organizations discussed in this manuscript: IFGworld. The researchers report no financial interest or executive relationships that could be construed as a potential conflict of interest.

Author names: Annabelle Ng and Maya Cratsley 
www.yumedtext.com | November-2019

\section{Funding}

This work was supported by Curing Childhood Cancer Foundation. The sponsor had no involvement with study design, collection, analysis, interpretation of data, writing of the report, or the decision to submit the article for publication.

\section{Acknowledgements}

The authors would like to thank Dr. Derek Suite, Dr. Stephen Liu, Dr. Earl Brien for providing input and assistance to this study. We would also like to thank the Curing Childhood Cancer Foundation for sponsoring this study.

\section{REFERENCES}

1. Cattell RB, Scheier IH. The meaning and measurement of neuroticism and anxiety. Oxford: Ronald, USA; 1961.

2. Cattell RB, Scheier IH. Handbook for the IPAT Anxiety Scale Questionnaire (self Analysis Form): A Brief, Valid, and Non-Stressful Questionnaire Scale, Measuring Anxiety Level in Adults and Young Adults Down to 14 Or 15 Years of Age. Illinois: Institute for Personality and Ability Testing, USA; 1963.

3. Spielberger CD. State-Trait anxiety inventory. In: Weiner IB, Craighead WE, editors. The Corsini encyclopedia of psychology. Hoboken: John Wiley \& Sons, Inc., USA; 2010. 1-1 p.

4. Appukuttan DP. Strategies to manage patients with dental anxiety and dental phobia: Literature review. Clin Cosmet Investig Dent. 2016;10(8):35-50.

5. White AM, Giblin L, Boyd LD. The prevalence of dental anxiety in dental practice settings. J Dent Hyg. 2017;91(1):30-4.

6. Lin CS, Wu SY, Yi CA. Association between anxiety and pain in dental treatment: A systematic review and meta-analysis. J Dent Res. 2016;96(2):153-62.

7. Navarro-Haro MV, Lopez-del-Hoyo Y, Campos D, et al. Meditation experts try virtual reality mindfulness: A pilot study evaluation of the feasibility and acceptability of virtual reality to facilitate mindfulness practice in people attending a mindfulness conference. PLoS One. 2017;12(11):e0187777.

8. Maples-Keller JL, Bunnell BE, Kim SJ, et al. The use of virtual reality technology in the treatment of anxiety and other psychiatric disorders. Harv Rev Psychiatry. 2017;25(3):103-13.

9. Roche K, Liu S, Siegel S. The effects of virtual reality on mental wellness: A literature review. Ment Health Fam Med. 2019;14:811-8.

10. Yu C, Lee H, Luo X. The effect of virtual reality forest and urban environments on physiological and psychological responses. Urban For Urban Green. 2018;35:106-14.

11. Riva G, Mantovani F, Capideville CS. Affective interactions using virtual reality: The link between presence and emotions. Cyberpsychol Behav. 2007;10(1):45-56. 
12. Niharika P, Reddy NV, Srujana P, et al. Effects of distraction using virtual reality technology on pain perception and anxiety levels in children during pulp therapy of primary molars. J Indian Soc Pedod Prev Dent. 2018;36(4):364-9.

13. Hoffman HG, Garcia-Palacios A, Patterson DR, et al. The effectiveness of virtual reality for dental pain control: A case study. Cyberpsychol Behav. 2001;4(4):527-35.

14. Wiederhold MD, Gao K, Wiederhold BK. Clinical use of virtual distraction system to reduce anxiety and pain in dental procedures. Cyberpsychol Behav Soc Netw. 2014;17(6):359-65.

15. Sweta VR, Abhinav RP, Ramesh A. Role of virtual reality in pain perception of patients following the administration of local anesthesia. Ann Maxillofac Surg. 2019;9(1):110-3.

16. Tanja-Dijkstra K, Pahl S, White MP, et al. Improving dental experiences by using virtual reality distraction: A simulation study. PLoS One. 2014;9(3):e91276.

17. Gujjar KR, Sharma R, Jongh AD. Virtual reality exposure therapy for treatment of dental phobia. Dent Update. 2017;44(5):423-35.

18. Gujjar KR, van Wijk A, Sharma R, et al. Virtual reality exposure therapy for the treatment of dental phobia: A controlled feasibility study. Behav Cogn Psychother. 2018;46(3):367-73.

19. Raghav K, van Wijk AJ, Abdullah F, et al. Efficacy of virtual reality exposure therapy for treatment of dental phobia: A randomized controlled trial. BMC Oral Health. 2016;16:25.

20. Chawla S, Li B, Liu S, et al. Let's relax! An immersion virtual reality relaxation intervention for quality of life improvement of cancer patients. J Neurology, Psych and Brain Res. 2018;1(3):1-7.

21. Liu M, Matsumura D. A Virtual Reality Relaxation Intervention on NCAA Division-One Student Athletes. EC Psychol Psychiatry. 2019;8(7):586-93.

22. Serbulea M, Payyappallimana U. Onsen (hot springs) in Japan-Transforming terrain into healing landscapes. Health Place. 2012;18(6):1366-73.

23. Yang B, Qin Q, Han L, et al. Spa therapy (balneotherapy) relieves mental stress, sleep disorder, and general health problems in sub-healthy people. Int J Biometeorol. 2018;62(2):261-72 